Relations industrielles

Industrial Relations

Manpower Issues in Manitoba : Report of the Manitoba

Economic Development Advisory Board, Winnipeg, Manitoba, June 1975.

\title{
Jean Sexton
}

Volume 31, numéro 1, 1976

URI : https://id.erudit.org/iderudit/028696ar

DOI : https://doi.org/10.7202/028696ar

Aller au sommaire du numéro

Éditeur(s)

Département des relations industrielles de l'Université Laval

ISSN

0034-379X (imprimé)

1703-8138 (numérique)

Découvrir la revue

Citer ce compte rendu

Sexton, J. (1976). Compte rendu de [Manpower Issues in Manitoba : Report of the Manitoba Economic Development Advisory Board, Winnipeg, Manitoba, June 1975.] Relations industrielles / Industrial Relations, 31(1), 160-161.

https://doi.org/10.7202/028696ar

Tous droits réservés (C) Département des relations industrielles de l'Université Laval, 1976
Ce document est protégé par la loi sur le droit d'auteur. L'utilisation des services d'Érudit (y compris la reproduction) est assujettie à sa politique d'utilisation que vous pouvez consulter en ligne.

https://apropos.erudit.org/fr/usagers/politique-dutilisation/ 
tion du texte a déjà consacré beaucuop d'énergies à analyser les relations du travail dans le secteur public de différents pays occidentaux. En effet, depuis 1968, douze monographies portant sur ce sujet ont déjà été publiées par l'Institute of Labor and Industrial Relations de l'Université du Michigan, la plupart sous la direction du professeur Rehmus.

L'analyse effectuée par chacun des auteurs nous révèle des considérations fort intéressantes sur chacun des pays et le lecteur peut en retirer les principales conclusions suivantes:

1- les deux pays nord-américains sont ceux où l'institutionnalisation de la négociation collective par les employés de l'État est la plus avancée. Dans tous les pays européens et même au Japon on semble attacher plus d'importance à l'incompatibilité de la relation contractuelle (convention collective) avec la relation statutaire traditionnelle (souveraineté de l'État). Le cas le plus évident est celui de l'Allemagne où l'on distingue très nettement les droits du "Beamte» (fonctionnaire) de ceux du «Arbeitnehmer» (employé couvert par le droit du travail). Cependant, il faut dire que cette incompatibilité est de plus en plus contestée et il n'est pas impossible de croire que d'ici quelques années elle ne disparaisse définitivement, le Japon étant sans doute le premier pays à évoluer dans cette direction.

2- Même dans les pays où la négociation collective des conditions de travail n'est guère avancée, il n'en demeure pas moins que les employés de l'État sont regroupés dans de puissantes associations capables de générer un «lobbying» efficace. Ces associations sont soit affiliées au reste du mouvement syndical, soit indépendantes; il n'y a pas d'uniformité làdessus d'un pays à l'autre.

3- L'inflation est un des problèmes communs à tous les pays et les tentatives d'en freiner le rythme ont causé de sérieux inconvénients aux employés de l'État. À chaque fois que les gouvernements occidentaux ont tenté d'imposer des contrôles de prix et de salaires, il s'est produit le phénomène suivant: l'État, pour être logique avec lui-même, imposait rigoureusement ses contrôles à ses employés. Par contre, le secteur privé de l'économie réussissait généralement à contourner les contrôles, de sorte que les conditions de travail des employés du secteur public se trouvaient relativement détériorées par rapport à celles des employés du secteur privé.
4- La question de la grève par les employés de l'État semble soulever beaucoup moins de problèmes dans les pays européens qu'en Amérique du Nord. Certains pays comme la Suède, la France et le Japon ont bien connu une certaine activité dans ce domaine, mais jamais dans les mêmes proportions que celle atteinte en Amérique du Nord depuis quelques années. Nous croyons que ce phénomène peut s'expliquer par l'absence, en Amérique du Nord, d'une tradition véritable dans le rôle joué par l'État dans l'économie et qui explique aussi sans doute pourquoi les mécanismes de détermination des conditions de travail du secteur privé ont été aussi facilement transposées dans le secteur public.

Public Employment Labor Relations: An Overview of Eleven Nations est un livre fort bien fait et qui ne manquera pas de plaire à tous ceux qui s'intéressent aux relations de travail dans le secteur public.

Université Laval

Jean BCIVIN

Manpower Issues in Manitoba: Report of the Manitoba Economic Development Advisory Board, Winnipeg, Manitoba, June 1975.

C'est suite à la conclusion que le Manitoba se dirigeait rapidement dans les années 1970 vers un problème de sous-emploi plutôt que vers un problème de chômage qui amena le Economic Development Advisory Board (E.D.A.B.) du Manitoba à faire examiner les problèmes de main-d'cuvre du Manitoba. C'est le résultat de cet examen que nous retrouvons dans ce rapport présenté en deux parties principales: 1 rapport et les recommandations du E.D.A.B. d'une part et neuf études spécifiques commanditées par le E.D.A.B. d'autre part. Examinons brièvement chacune de ces deux parties.

Quant au rapport de l'E.D.A.B., il part de l'observation, fort juste d'ailleurs, que la politique de main-d'œuvre au Manitoba est encore au stage du balbutiement et qu'une telle politique de main-d'œuvre doit viser à corriger le marché du travail. Cette double constatation a amené l'E.D.A.B. à faire un exercice pour connaître les défauts du o des marchés du travail manitobain(s) pour ensuite bâtir des correcteurs valables qui répondent exactement aux besoins de cette province.

Un tel exercice enseigne au moins deux leçons: d'abord il refère à la nécessité de bien diagnostiquer l'état du marché du travail avant de faire une politique et avant 
de structurer des programmes ou services de main-d'œuvre. Le Québec aurait présentement grand intérêt à suivre l'exemple du Manitoba. En second lieu, un tel exercice indique clairement l'orientation que prendra la politique de main-d'œuvre dans cette province: corriger le marché du travail. Même si cette leçon peut sembler évidente à plusieurs, elle est d'autant plus utile qu'elle sert à identifier clairement la mission et le champ d'action propre à une telle politique publique et peut servir à limiter les problèmes de juridiction entre ministères de même juridiction dans le domaine de la main-d'œuvre.

Le comité fait au total huit recommandations très générales qui frisent souvent le vœu pieux et qui touchent plusieurs aspects d'une politique de main-d'œuvre (P.M.O.). Cependant ces huit recommandations montrent clairement que le E.D.A.B. n'a pas encore une idée claire et bien structurée de ce qu'est une véritable P.M.O. du moins théoriquement. On a aboutit alors à identifier la principale faiblesse de ce rapport: l'absence d'une conception claire et d'un cadre de référence structuré sur la P.M.O.

Quant à la seconde partie de ce rapport, on y retrouve neuf études portant sur certains aspects économiques très particuliers du marché du travail. Ces études, généralement bien faites, représentent un effort d'exploration utile à l'élaboration d'une P.M.O. Cependant, un tel effort devra se poursuivre pour en arriver à définir une P.M.O. pour le Manitoba.

En résumé, cet ouvrage est intéressant à plusieurs égards. Il montre l'effort d'une province à se définir une P.M.O. propre à ses besoins. Cet effort, qui s'inscrit dans le mouvement de provincialisation de la P.M.O. au Canada, n'est cependant pas complet. Un peut neanmoins retirer des leçons utiles autant des aspects forts que des carences de ce rapport.

Jean SEXTON

Université Laval

Insécurité d'emploi et relations de travail dans l'industrie canadienne de la construction, par Paul Malles, Ottawa, Conseil économique du Canada, 1975, $122 \mathrm{pp}$.

Cet ouvrage de Paul Malles constitue un document préparatoire au rapport du Conseil économique du Canada, rapport portant sur l'instabilité dans l'industrie de la construction et intitulé: "Pour une croissance plus stable de la construction».
Cette étude se penche plus particulièrement sur l'effet de l'instabilité sur les relations du travail dans l'industrie canadienne de la construction.

L'industrie de la construction est très différente des autres industries. À preuve, bien que n'employant que $6 \%$ de la maind'œuvre, elle compte pour $19 \%$ des jourshomme perdus. Dans le chapitre consacré aux facteurs d'instabilité, l'auteur insiste sur l'importance des effets secondaires des conflits du travail à l'intérieur de l'industrie de la construction. Cette importance des effets secondaires est une autre différence entre l'industrie de la construction et les autres. Un autre facteur d'instabilité est celui de la disparité salariale existant entre le travailleur de l'industrie de la construction et celui des autres industries.

Un aspect qui ressort tout au long d l'ouvrage, est celui du statut particulier que détient le Québec en regard des autres provinces. Ces différences seront perçues aussi bien du point de vue juridique (bill 290 , décret de la construction, loi 9) que syndical (rivalité syndicale).

L'auteur parle des syndicats ouvriers de la construction. Il est intéressant de souligner le pouvoir conservé par les sections locales dans les syndicats internationaux de la construction ainsi que le peu de rapport entre celles-ci. Ce n'est que dernièrement qu'est apparu le Comité consultatif pour les métiers de la construction au Canada. Les associations patronales ont l'originalité d'être beaucoup plus organisées que celles des autres industries avec l'Association canadienne de la construction (ACC) et ajoutons, au Québec, avec la toute nouvelle Association des entrepreneurs en construction du Québec.

Un chapitre entier est consacré à la législation concernant les relations du travail. L'auteur présente sommairement les différentes législations dans le domaine de la construction et ce pour chaque province du Canada. Dans ce même chapitre, une attention particulière est portée au «système de décrets » de la province de Québec.

L'auteur présente également tout au long d'un chapitre les avantages et les inconvénients de la centralisation des relations du travail à laquelle il attribue comme inconvénients majeurs la multiplication des conflits et la présence de grèves illégales ou «sauvages».

Le type de conventions collectives de la construction est analysé dans un chapitre de cet ouvrage. On y constate par exemple la tendance pour la province d'Ontario à 